\title{
Weak Limits of the Quantum Hydrodynamic Model
}

\author{
PAOLA PIETRA $^{\mathrm{a}, *}$ and CARSTEN POHL ${ }^{\mathrm{b}, \dagger}$ \\ ${ }^{a}$ Istituto di Analisi Numerica del C.N.R., Via Abbiategrasso 209, I-27100 Pavia, Italy; ${ }^{\mathrm{b}}$ Johannes Kepler Universität Linz, \\ Institut für Analysis und Numerik, Abtl. Differentialgleichungen, Altenberger Str. 69, A-4040 Linz, Austria
}

(Received 13 August 1997; Revised 1 December 1998)

\begin{abstract}
A numerical study of the dispersive limit of the quantum hydrodynamic equations for semiconductors is presented. The solution may develop high frequency oscillations when the scaled Planck constant is small. Numerical evidence is given of the fact that in such cases the solution does not converge to the solution of the formal limit equations.
\end{abstract}

Keywords: Quantum hydrodynamic model, dispersive limit

\section{INTRODUCTION}

Semiconductor models based on classical or semi-classical mechanics (like the drift-diffusion equation, the hydrodynamic models and the semiclassical solid state physics Boltzmann equation) cannot be used to reasonably describe the performance of ultra-integrated devices, which are based on quantum effects. Typical examples for such devices are resonant tunneling diodes [5]. Recently the so-called quantum hydrodynamic model (QHD) has been introduced (see e.g. $[2,5,10]$ ). The QHD model has the advantage of dealing with macroscopic fluid-type unknowns (and consequently fluid-type boundary conditions can be incorporated) and it is able to describe quantum phenomena, such as negative differential resistance in a resonant tunneling diode.
Mathematically, the QHD system is a dispersive regularization of the hydrodynamic equations (HD). The regularization depends on the scaled Planck constant $\varepsilon$ and, formally, vanishes in the classical limit $\varepsilon \rightarrow 0$. Thus, in the formal limit, the QHD model tends to the HD model. However, due to the non-linearity and the dispersive nature of the regularization, one cannot expect that the formal limit describes the correct limiting behavior in general. Due to the dispersive term, the solutions of the QHD system may develop fast oscillations which are not damped as $\varepsilon$ goes to zero and in that case the limiting system is not expected to be the HD system.

Here we present numerical evidence of the fact that the solution of the QHD system develops dispersive oscillations when the corresponding HD system exhibits a shock wave and that the weak

\footnotetext{
*Tel.: + 39 (0) 382 505690, e-mail: pietra@dragon.ian.pv.cnr.it

${ }^{\dagger}$ Corresponding author. Tel.: +43 (0) 732-2468 9187, e-mail: carsten.pohl@jk.uni-linz.ac.at
} 
limit of the QHD solution is not a solution of the HD equations. The understanding of the dispersive nature of the problem and of its dispersive limit is an important issue in semiconductor applications, since the scaled Planck constant $\varepsilon$ is often small $\left(\varepsilon \sim 10^{-2}, 10^{-3}\right)$. In particular, it gives important hints on the choice of reliable numerical schemes.

The paper is organized as follows. Section 2 presents the derivation of the isothermal/isentropic QHD equations from the Schrödinger equation. Section 3 collects known results about a dispersive model problem (Korteweg-de Vries equation) and compares two low order space discretization schemes. Section 4 is devoted to rigorous (partial) results for the QHD model in the literature. Finally, the numerical study of the dispersive limit for the isothermal QHD system is presented in Section 5.

\section{QHD EQUATIONS: DERIVATION}

There are different ways to derive the QHD equations. Here we present the simple derivation based on a quantum mechanical pure state described by a non-linear Schrödinger equation [13].

We consider the (scaled) Schrödinger equation

$$
i \varepsilon \psi_{t}=-\frac{\varepsilon^{2}}{2} \Delta \psi+\left(h\left(|\psi|^{2}\right)+V\right) \psi, \quad x \in \mathbb{R}^{d}, t \in \mathbb{R}
$$

subject to the initial condition

$$
\psi(x, t=0)=\psi_{I}(x), \quad x \in \mathbb{R}^{d} .
$$

$\psi$ denotes the wave function of the pure state and $\varepsilon$ the scaled Planck constant. The function $h(s)$ stands for the enthalpy (representing the multiparticle physics). The potential $V$ satisfies the selfconsistently coupled Poisson equation

$$
-\lambda^{2} \Delta V=n-C(x), \quad x \in \mathbb{R}^{d}
$$

where $n=|\psi|^{2}$ is the particle density and $\lambda$ is the scaled Debye length. In semiconductor applications $C(x)$ denotes the given doping profile.

By using the WKB-Ansatz

$$
\psi(x, t)=\sqrt{n(x, t)} e^{\frac{1}{\varepsilon} S(x, t)}
$$

( $S$ is the scaled phase of the wave function), and separating the real and imaginary parts in the Schrödinger equation, we obtain the following irrotational flow equations

$$
n_{t}+\operatorname{div}(n \nabla S)=0
$$

and

$$
S_{t}+\frac{1}{2}|\nabla S|^{2}+V+h(n)-\frac{\varepsilon^{2}}{2}\left(\frac{1}{\sqrt{n}} \Delta \sqrt{n}\right)=0 .
$$

The definition of the current density

$$
J(x, t)=\varepsilon \operatorname{Im}(\overline{\psi(x, t)} \nabla \psi(x, t))
$$

gives

$$
J=n \nabla S .
$$

Taking the gradient of (2.6), multiplying by $n$ and using (2.5), we obtain the QHD flow equations for the density $n$ and current density $J$ coupled to the Poisson equation (2.3)

$$
n_{t}+\operatorname{div} J=0
$$

$$
\begin{aligned}
J_{t} & +\operatorname{div}\left(\frac{J \otimes J}{n}+p(n)\right) \\
& +n \nabla V-\frac{\varepsilon^{2}}{2} n \nabla\left(\frac{\Delta \sqrt{n}}{\sqrt{n}}\right)=0
\end{aligned}
$$

( $\otimes$ denotes the tensor product of vectors). The density dependent pressure $p(n)$ and the enthalpy $h(n)$ are related by

$$
p^{\prime}(n)=n h^{\prime}(n) .
$$

The isothermal case corresponds to $p(n)=n$ (and $h(n)=\log (n))$, and the isentropic case corresponds to $p(n)=n^{\gamma}, 1<\gamma<3\left(\right.$ and $\left.h(n)=\frac{\gamma}{\gamma-1} n^{\gamma-1}\right)$. 
In order to model the influence of collisions between electrons and the lattice atoms the relaxation term

$$
-\frac{J}{\tau}
$$

with a small relaxation time $\tau$, is added to the right hand side of Eq. (2.8b). It is obtained with purely phenomenological considerations and cannot be deduced from the Schrödinger equation.

In the Wigner-Boltzmann derivation of QHD models, the relaxation term comes from a FokkerPlanck relaxation approximation of the collision term. This is discussed in [11].

\section{KORTEWEG-DE VRIES EQUATION}

The Korteweg-de Vries (KdV) equation is the simplest model problem for dispersive phenomena.

$$
\begin{gathered}
u_{t}^{\varepsilon}+u^{\varepsilon} u_{x}^{\varepsilon}+\varepsilon^{2} u_{x x x}^{\varepsilon}=0, \quad x \in \mathbb{R}, \quad t \in \mathbb{R} \\
u^{\varepsilon}(x, t=0)=u_{I}, \quad x \in \mathbb{R} .
\end{gathered}
$$

The Korteweg-de Vries equation is a dispersive approximation of the non-linear hyperbolic Burgers' (B) equation with the same initial function $u_{I}$

$$
\begin{array}{r}
u_{t}+u u_{x}=0, \quad x \in \mathbb{R}, \quad t \in \mathbb{R} \\
u(x, t=0)=u_{I}, \quad x \in \mathbb{R} .
\end{array}
$$

The small dispersion limit of the $\mathrm{KdV}$ equation has been studied in $[12,19]$ using the complete integrability of $\mathrm{KdV}$. As long as (B) has a smooth solution, the $\mathrm{KdV}$ solution $u^{\varepsilon}(x, t)$ tends strongly to that smooth solution. However, when $t$ exceeds the time where the smooth solution of (B) breaks down, $u^{\varepsilon}(x, t)$ develops dispersive oscillations. More precisely, as $\varepsilon$ tends to zero, the amplitude of these oscillations remains bounded but does not tend to zero, and its wave length is of order $\varepsilon$. The convergence of $u^{\varepsilon}(x, t)$ to a limit then holds only weakly. Let us denote the weak limit of $u^{\varepsilon}(x, t)$ by $u^{0}$. Clearly, since the weak limit of $\left(u^{\varepsilon}\right)^{2}$ in general is not the square of the weak limit of $u^{\varepsilon}, u^{0}$ is not a weak solution of $(B)$.

This result is illustrated in Figure 1. Equation (3.1) is solved on the interval $(0,2 \pi)$ with periodic boundary conditions and a positive periodic initial condition $\left(u_{I}(x)=\cos (x)+1.5\right)$. With this choice of $u_{I}$ the Burgers' equation develops a shock at time $t=1$. The solutions of (B) (solid line) and of $\mathrm{KdV}$ for two values of $\varepsilon$ (dashed line: $\varepsilon=0.1$; dashed-dotted line: $\varepsilon=0.05$ ) are shown at time $t=2.51$. The dispersive oscillations are clearly present (the amplitude of the oscillations does not change as $\varepsilon$ goes to zero and the frequency doubles as $\varepsilon$ is halved). Moreover, since the oscillations go beyond the shock of $u$, it is evident that the weak limit of the $\mathrm{KdV}$ solution is not the entropy solution of (B).

A generalized $\mathrm{KdV}$ equation is considered in $[3,17]$

$$
\begin{gathered}
u_{t}^{\varepsilon, \delta}+u^{\varepsilon, \delta} u_{x}^{\varepsilon, \delta}+\varepsilon^{2} u_{x x x}^{\varepsilon, \delta}=\delta u_{x x}^{\varepsilon, \delta}, \quad x \in \mathbb{R}, \quad t \in \mathbb{R} \\
u^{\varepsilon, \delta}(x, t=0)=u_{I}, \quad x \in \mathbb{R} .
\end{gathered}
$$

In this case, the presence of the dissipative term $\delta u_{x x}$ requires an accurate account for the relative sizes of $\delta$ and $\varepsilon$. When the dispersive term is absent $(\varepsilon=0)$, the solution of (3.3) tends, as $\delta$ goes to zero, to the entropy solution of (B). When both dispersive and dissipative terms are present, oscillations occur but the weak limit is the entropy solution of (B) if diffusion dominates $(\delta \geq$ const $\varepsilon$ ).

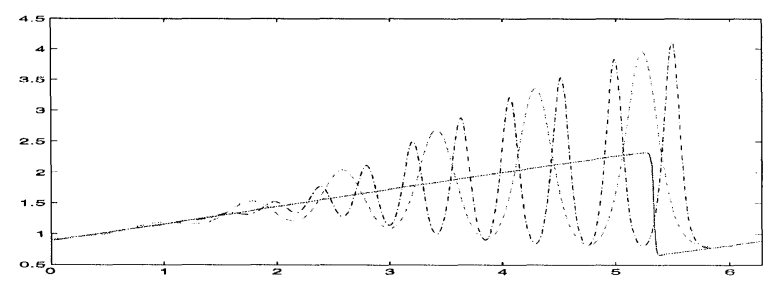

FIGURE 1 Solution of (B) and solutions of $\mathrm{KdV}$ for $\varepsilon=0.1$ and $\varepsilon=0.05$. 
Only when dispersion dominates, the weak limit is not a solution of the (B) equation.

It is clear that the choice of a discretization scheme for $\mathrm{KdV}$ must take into account the nature of the dispersive character of the equation. Looking at $\mathrm{KdV}$ as a perturbation of the Burgers' equation can be misleading. A numerical scheme of hyperbolic type for the first order term can easily give wrong results, unless the space mesh size is very small. A scheme for the Burgers' equation which guarantees convergence to the entropy solution contains a diffusion term with a diffusion coefficient going to zero with the mesh size. For instance, the classical upwind scheme introduces an artificial viscosity term of the order of the mesh size. Due to the presence of numerical viscosity, the computed solution may fail to exhibit fundamental properties of the true solution, unless the mesh size is much smaller than $\varepsilon$.

When a standard central difference scheme is used for the first order term, no numerical dissipation is introduced. The leading term of the Taylor expansion of the truncation error is $\frac{u\left(x_{i+1}\right)-u\left(x_{t-1}\right)}{2 h}-u_{x}\left(x_{i}\right) \simeq \frac{1}{6} h^{2} u_{x x x}$ and smoothing of the oscillations is not expected.

Figure 2 compares the solution of (B) as in Figure 1 (solid line), with the solution of $\mathrm{KdV}$ for $\varepsilon=0.05$ computed using the first order upwind scheme and the standard 5 point second order formula for $u_{x x x}$. In order to focus only on the effects of the space discretization, a Crank-Nicolson scheme with a very small time step $\Delta t$ is used to advance in time. The dotted line corresponds to 320 nodes, the dashed line is obtained with 640 nodes and the dashed-dotted line corresponds to

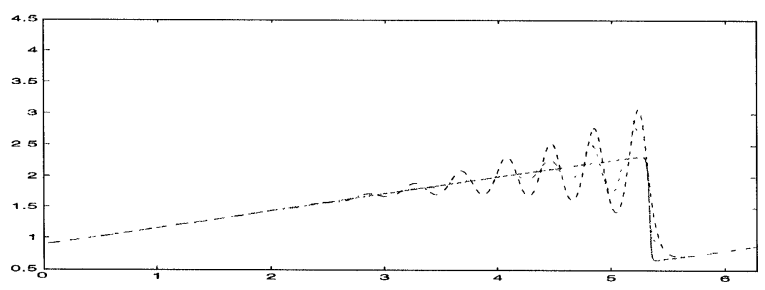

FIGURE 2 Solution of (B) and solutions of $\mathrm{KdV}$ with an upwind scheme, $\varepsilon=0.05$, various number of nodes.

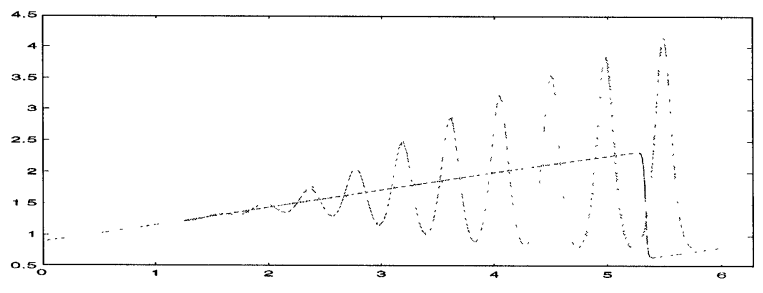

FIGURE 3 Solution of (B) and solutions of $\mathrm{KdV}$ with central differences, $\varepsilon=0.05,320$ and 640 nodes.

1280 nodes. Clearly the computed solution suffers from strong dissipation and even the 1280 node case gives a solution which is an approximation of the (B) equation (the oscillations have smaller amplitude, compared to the solution in Figure 1 and the oscillation with the largest amplitude is placed at the shock of $u$ ). When the upwind scheme is replaced by the standard two point central finite difference approximation, many fewer nodes are necessary to compute the solution with good accuracy. Figure 3 shows the solution of $\mathrm{KdV}$ with the central finite difference scheme for $\varepsilon=0.05$ and 320 nodes (solid line) and for 640 nodes (dotted line).

For high order approximation schemes we refer to [4], where a pseudospectral discretization is proposed and analyzed also in the case of a small dispersion parameter $\varepsilon$.

\section{WEAK LIMITS}

The small dispersion limit of the QHD system is an open problem. Only partial answers are known in special cases $[6,9]$. These results are given in the context of the Schrödinger equation and they apply to QHD-type problems without relaxation term. Moreover, WKB initial conditions with $n_{I}, J_{I}$ independent of $\varepsilon$ are considered for the Schrödinger equation in those references. Hence the initial flow of the QHD system is irrotational. In the following, we denote by $n^{0}$ and $J^{0}$ the weak limits of $n^{\varepsilon}$ and $J^{\varepsilon}$, solution of (2.8).

A complete characterization of $n^{0}$ and $J^{0}$ is given in [6] only in the constant pressure case with prescribed regular potential $V$. The starting point 
is the construction of the Wigner equation equivalent to the single state Schrödinger equation and the rigorous passage to the limit of the Wigner function to the Wigner measure. The (weak) limits of $n^{\varepsilon}$ and $J^{\varepsilon}$ as $\varepsilon \rightarrow 0$ can then be completely characterized using the Wigner measure theory ([14, $15,7])$. The Wigner $w^{0}=w^{0}(x, v, t)$ of the wave function $\psi^{\varepsilon}(t)$ is the solution of the Liouville equation,

$$
\begin{aligned}
\frac{\partial}{\partial t} w^{0}(x, v, t)+ & v \cdot \nabla_{x} w^{0}(x, v, t) \\
& -\nabla_{x} V(x) \cdot \nabla_{v} w^{0}(x, v, t)=0
\end{aligned}
$$

subject to the initial condition

$$
w_{I}^{0}(x, v)=n_{I}(x) \delta\left(v-\nabla S_{I}(x)\right),
$$

(where $\delta$ denotes the Dirac measure). Then, conservation of energy gives

$$
n^{\varepsilon} \rightarrow n^{0}:=\int_{\mathbb{R}_{v}^{m}} w^{0}(x, d v, t),
$$

and

$$
J^{\varepsilon} \rightarrow J^{0}:=\int_{\mathbb{R}_{v}^{m}} v w^{0}(x, d v, t) .
$$

The formal limit equation of QHD in this particular case is the pressureless Euler equation with forcing due to the electrostatic potential

$$
\begin{gathered}
n_{t}+\operatorname{div}(n u)=0 \\
u_{t}+\frac{1}{2}(u \cdot \nabla) u+\nabla V=0 \\
n(x, t=0)=n_{I}(x), \quad u(x, t=0)=\nabla S_{I}(x)
\end{gathered}
$$

where $u$ denotes the mean velocity and $J=n u$. Analogously to the $\mathrm{KdV}$ case illustrated in the previous section, as long as the solution of the field driven Burgers' equation (4.3b) is smooth, $n^{0}$ and
$J^{0}$ are solution of the system (4.3). However, after the breakdown of the classical solution of the Burgers' equation (4.3b), $n^{0}$ and $J^{0}$ develop singularities and they are not anymore solutions of (4.3).

In [9] a nonlinear Schrödinger equation (without self-consistent potential) is considered. Convergence to the solution of the hyperbolic limit problem is proved as long as the solution of the isentropic HD (Euler) system is smooth. After the breakdown, the weak limits are not identified.

\section{NUMERICAL STUDY}

Here we present a numerical study of the weak limits of the isothermal, stationary, one dimensional quantum hydrodynamic equations

$$
J_{x}^{\varepsilon}=0
$$

$$
\left(\frac{\left(J^{\varepsilon}\right)^{2}}{n^{\varepsilon}}+n^{\varepsilon}\right)_{x}+n^{\varepsilon} V_{x}-\frac{\varepsilon^{2}}{2} n^{\varepsilon}\left(\frac{\sqrt{n^{\varepsilon}} x x}{\sqrt{n^{\varepsilon}}}\right)_{x}=-\frac{J^{\varepsilon}}{\tau}
$$

$$
-\lambda^{2} \Delta V=n^{\varepsilon}-C(x)
$$

with $x \in(0,1)$. As boundary conditions we used:

$$
\begin{gathered}
n^{\varepsilon}(0)=C(0), \quad n^{\varepsilon}(1)=C(1), \quad n_{x}^{\varepsilon}(0)=0=n_{x}^{\varepsilon}(1) \\
V(0)=V_{0}, \quad V(1)=0 .
\end{gathered}
$$

As pointed out before, the Eqs. (5.1) are a dispersive regularization of the classical isothermal hydrodynamic equations

$$
J_{x}=0
$$

$$
\begin{gathered}
\left(\frac{J^{2}}{n}+n\right)_{x}+n V_{x}=-\frac{J}{\tau} \\
-\lambda^{2} \Delta V=n-C(x) .
\end{gathered}
$$


It is well known that system (5.2) can develop a shock discontinuity in the transition from a supersonic region to a subsonic region, while the transition from the subsonic to the supersonic regime is smooth (see e.g. [1]). The sound speed is $c=1$, thus, $J<n$ characterizes the subsonic zone, and $J>n$ characterizes the supersonic zone (we recall that the velocity is $u=J / n$ ). With asymptotic analysis arguments, it is possible to show that the QHD system (5.1) may develop dispersive oscillations with $\varepsilon$ wave length in the supersonic zone and it does note have dispersive oscillations in the subsonic region. Both transitions (subsonic to supersonic, and supersonic to subsonic) are not fully understood yet. We refer to [18] and, for a numerical study, to [8].

In order to avoid numerical damping of the oscillations, system (5.1) is discretized with a central finite difference scheme. A damped Newton algorithm is then used to solve the non-linear discrete problem. We refer to [16] for details.

In the numerical tests presented here, a $n^{+} n n^{+}$ diode is considered, with the smooth doping profile $C$ plotted in Figure $4(\max (C)=1, \min (C)=$ $0.1)$. The Debye length is chosen $\lambda=0.1$, the relaxation time $\tau=1 / 8$, and the applied voltage $V_{0}=6.5$.

In Figure 5 we present the solution of the hydrodynamic equations (5.2). The density $n$ has a shock discontinuity (approximately at $x=0.7$ ). The horizontal line is the (constant) current $J$. The shock occurs in the transition from the supersonic region $(J>n)$ to the subsonic region $(J<n)$.

Figures 6-9 show the solutions $n^{\varepsilon}$ and $J^{\varepsilon}$ of (5.1) for various values of $\varepsilon(\varepsilon=0.01, \varepsilon=0.005$, $\varepsilon=0.0026, \varepsilon=0.001$, respectively). Figure 10 is a zoom of Figure 9 in the neighbourhood of the

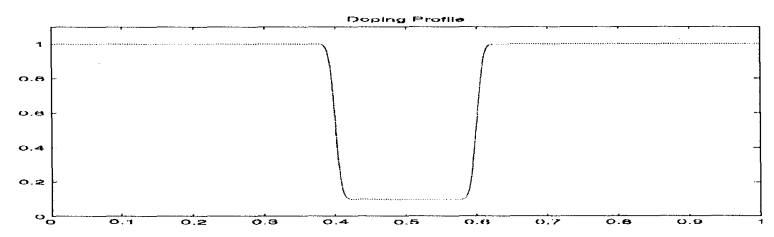

FIGURE 4 Doping profile $C$.

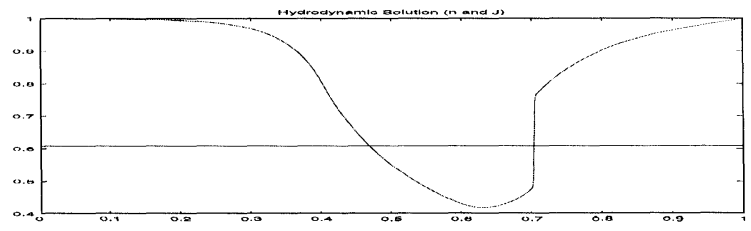

FIGURE 5 Solution of the hydrodynamic equations.

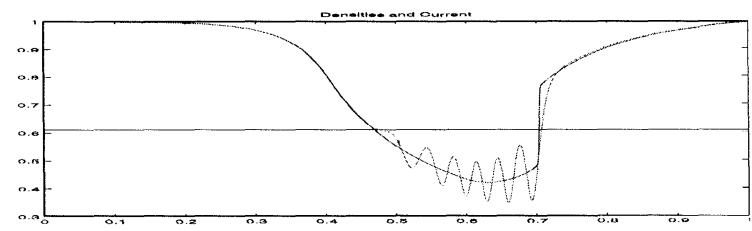

FIGURE 6 Solution for $\varepsilon=0.01$ (QHD and HD).

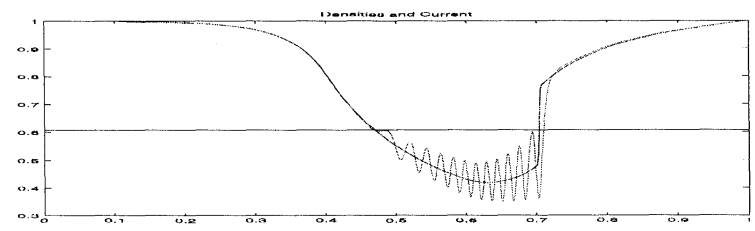

FIGURE 7 Solution for $\varepsilon=0.005$ (QHD and HD).

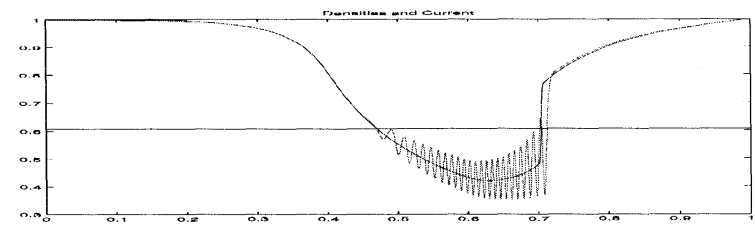

FIGURE 8 Solution for $\varepsilon=0.0026$ (QHD and HD).

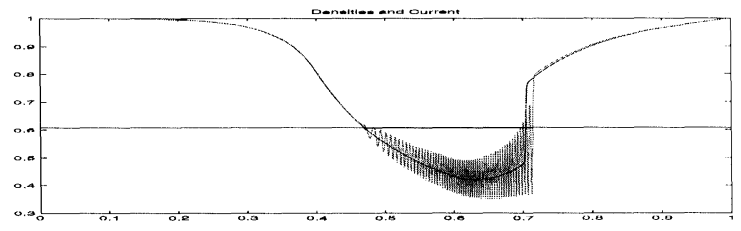

FIGURE 9 Solution for $\varepsilon=0.001$ (QHD and HD).

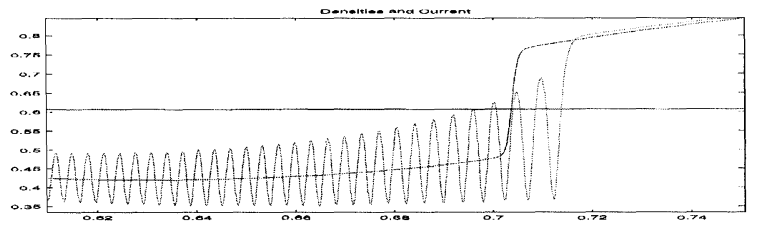

FIGURE 10 Solution for $\varepsilon=0.001$ (QHD and HD) (Zoom). 


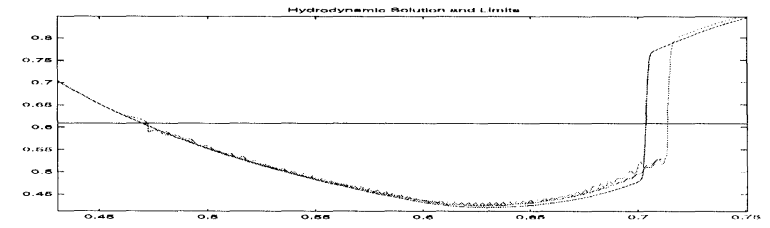

FIGURE 11 Weak limits and HD solution (Zoom).

shock discountinuity. For reference purposes also the solution of the hydrodynamic equation is plotted. The high frequency oscillations show a typical dispersive behaviour: the wavelength is of order $\varepsilon$ and the amplitude is of order 1. Moreover, as in the $\mathrm{KdV}$ case presented in Section 3, the oscillations of $n^{\varepsilon}$, solution of (5.1), go beyond the shock discontinuity of $n$, solution of (5.2), and it is evident that the weak limit of $n^{\varepsilon}$ as $\varepsilon \rightarrow 0$ (denoted by $n^{0}$ ) is not equal to $n$. Figure 11 illustrates the situation. The HD solution is plotted, together with two curves, which are obtained by taking mean values (in two different ways) over the oscillations. They can be considered as good approximations of $n^{0}$. A comparison between Figures 6-9 and Figure 11 suggests a conjecture on the behaviour of the QHD solution and its weak limit in the transonic transitions. The subsonicsupersonic transition, smooth in the HD case, occurs in a regular manner also in the QHD case. The oscillations do not perturb that subsonic region and the weak limit $n^{0}$ seems to coincide with the HD solution $n$ before the transition. The situation is more intricate for the supersonic-subsonic transition. For small $\varepsilon$ 's, (see Fig. 8 and Fig. 9), some large amplitude oscillations become partly subsonic, however the oscillations are confined in the supersonic region of the weak limit $n^{0}$ (see Fig. 11). The supersonic-subsonic transition point of $n$ and of $n^{0}$ are different. Moreover, the effect of the dispersive oscillations is transmitted to the subsonic zone. The weak limit $n^{0}$ differs from the HD solution in the supersonic region and in the subsonic region after that transition.

\section{Acknowledgements}

Both authors acknowledge support from the DAAD-VIGONI. The first author was also supported by the CNR-Progetto Speciale 96 "Mathematical models for semiconductors" and the second author by the DFG-project \#MA 1662/1-2.

\section{References}

[1] Ascher, U., Markowich, P. A., Pietra, P. and Schmeiser, C. (1991). A Phase Plane Analysis of Transonic Solutions for the Hydrodynamic Semiconductor Model. Math. Mod. and Meth. in the Appl. Sci., 1, 347-376.

[2] Ancona, M. G. and Iafrate, G. J. (1989). Quantum correction to the equation of state of an electron gas in a semiconductor. Phys. Rev., B39, 9536-9540.

[3] Forest, M. G. and McLaughlin, D. W. (1984). Modulations of perturbed KdV wavetrains. SIAM J. Appl. Math., 44, $287-300$

[4] Fornberg, B. and Whitham, G. B. (1978). A numerical and theoretical study of certain non-linear wave phenomena. Proc. Roy. Soc. London, 289, 373-404.

[5] Gardner, C. L. (1994). The quantum hydrodynamic model for semiconductor devices. SIAM J. Appl. Math., 54, $409-427$.

[6] Gasser, I. and Markowich, P. A. (1997). Quantum hydrodynamics, Wigner transforms and the classical limit. Asymptotic Analysis, 14, 97-116.

[7] Gérard, P., Markowich, P. A., Mauser, N. J. and Poupaud, F. (1997). Homogenization Limits and Wigner Transforms. Comm. Pure and Appl. Math., 50, 321-377.

[8] Gasser, I., Markowich, P. A., Schmidt, D. and Unterreiter, A. (1995). Macroscopic Theory of Charged Quantum Fluids, In: Mathematical Problems in Semiconductor Physics, Marcati, P., Markowich, P. A. and Natalini, R. (Eds.), Pitman Research Notes in Mathematics Series 340 , Longman, pp. $42-75$.

[9] Grenier, E. (1995). Limite semi-classique de l'equation de Schrödinger non linaire en temps petit. C.R. Acad. Sci. Paris Sr. I Math., 320(6), 691-694.

[10] Gardner, C. L. and Ringhofer, C. (1998). Smooth Quantum Hydrodynamic Model Simulation of the Resonant Tunneling Diode. To appear in VLSI Design.

[11] Gardner, C. L. and Ringhofer, C. (1996). Smooth Quantum Potential for the Hydrodynamic Model. Physical Review E, 53, 157-167.

[12] Lax, P. D. and Levermore, C. D. (1983). The small dispersion limit of the KdV equation. Comm. Pure Appl. Math., 36, I 253-290; II 571-593; III 809-830.

[13] Landau, L. D. and Lifschitz, E. M. (1985). Lehrbuch der Theoretischen Physik III-Quantenmechanik. AkademieVerlag.

[14] Lions, P. L. and Paul, T. (1993). Sur les Mesures de Wigner. Revista Mat. Iberoamericana, 9, 553-618.

[15] Markowich, P. A. and Mauser, N. J. (1993). The Classical Limit of a Self-consistent Quantum-Vlasov Equation in 3-D. Math. Mod. and Meth. in Appl. Sciences, 9, 109-124. 
[16] Pietra, P. and Pohl, C., A numerical Study of the isentropic Quantum Hydrodynamic Equation for Semiconductors. in preparation

[17] Schonbek, M. E. (1982). Convergence of solutions to nonlinear dispersive equations. Comm. Partial Differ. Eq., 7, 959- 1000 .

[18] Schmeiser, C., in preparation

[19] Venakides, S. (1987). The zero dispersion limit of the periodic KdV equations. AMS Transaction, 301, 189-226.

\section{Authors' Biographies}

Paola Pietra Born on 30.9.1955 in Pavia, Studies of Mathematics at the Università di Pavia: 19741978, Position: Research Director, Professional career: C.N.R. fellowship at the Istituto di Analisi Numerica, Pavia, 1979-1982, Researcher at the same institution, $1982-1991$, Research Director at the same institution from 1991 to now, Postdoctoral position at the Department of Mathematics of the University of Chicago from September 1984 to October 1985, Visiting Associate Professor at the Mathematics Department of Purdue University, West Lafayette, Indiana, from August 1990 to May 1991.

Carsten Pohl Born on the 25.5.1968 in Berlin. October 1987-April 1994: Studies of mathematics at the TU Berlin, masters exam with distinction, May 1994-May 1998: PhD student at the TU Berlin, since May 1998 at the Johannes Kepler University Linz, June 1998: PhD exam with distinction. 

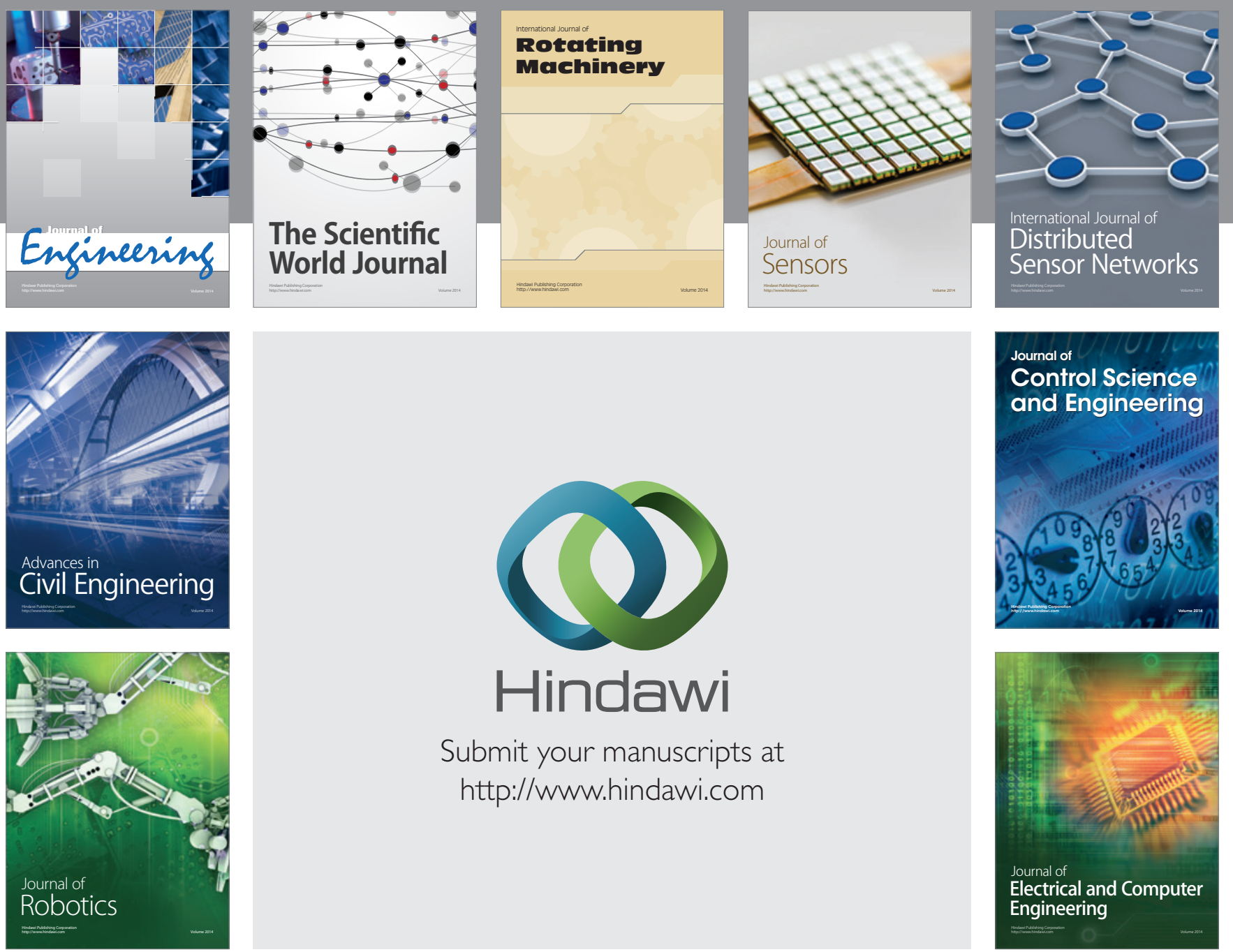

Submit your manuscripts at

http://www.hindawi.com
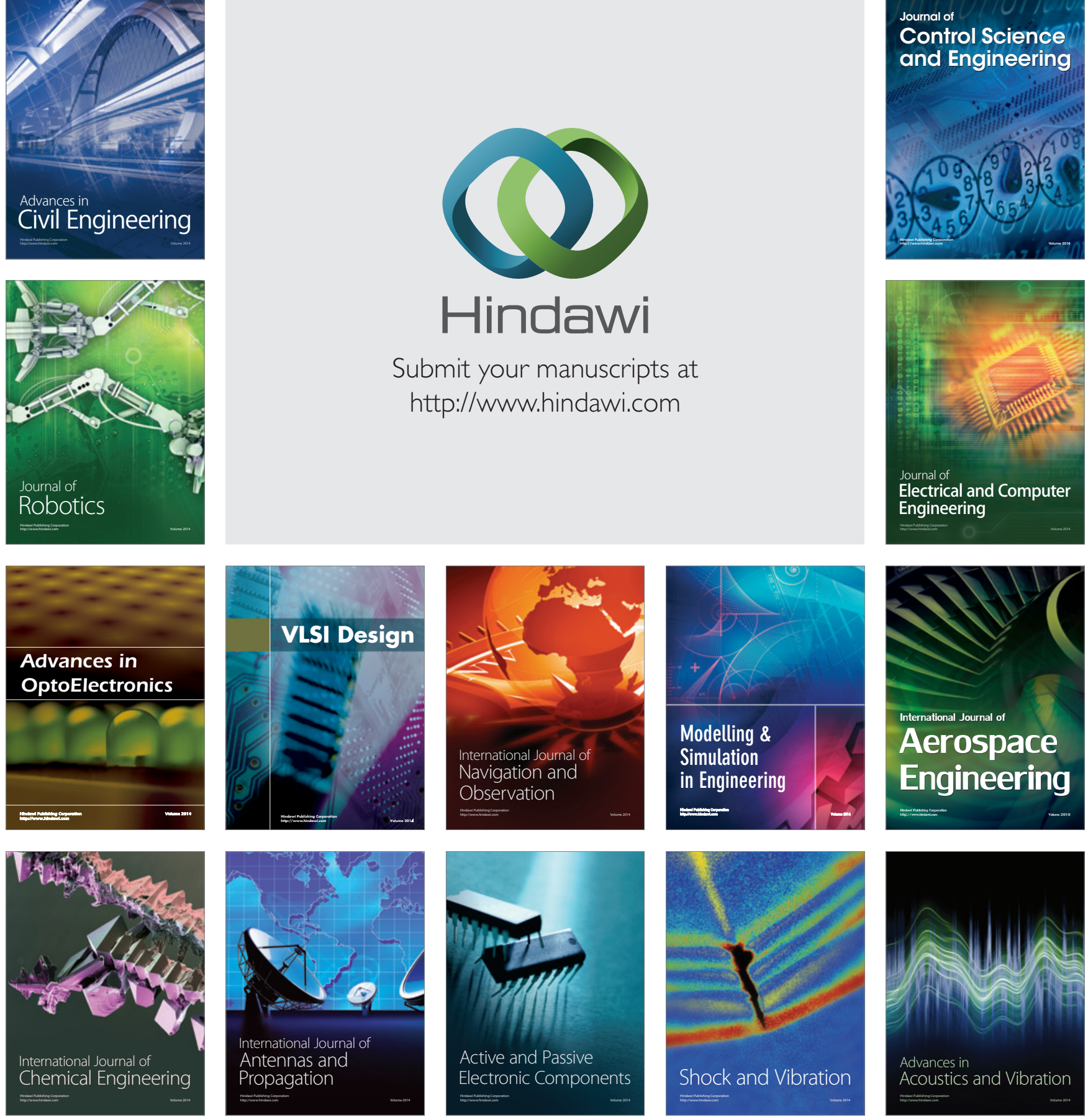